\title{
Nanostructured Surfaces to Target and Kill Circulating Tumor Cells While Repelling Leukocytes
}

\author{
Michael J. Mitchell, Carlos A. Castellanos, and Michael R. King \\ Department of Biomedical Engineering, Cornell University, Ithaca, NY 14853, USA \\ Correspondence should be addressed to Michael R. King, mike.king@cornell.edu
}

Received 19 November 2012; Accepted 5 December 2012

Academic Editor: Haiyan Li

Copyright ( $) 2012$ Michael J. Mitchell et al. This is an open access article distributed under the Creative Commons Attribution License, which permits unrestricted use, distribution, and reproduction in any medium, provided the original work is properly cited.

\begin{abstract}
Hematogenous metastasis, the process of cancer cell migration from a primary to distal location via the bloodstream, typically leads to a poor patient prognosis. Selectin proteins hold promise in delivering drug-containing nanocarriers to circulating tumor cells (CTCs) in the bloodstream, due to their rapid, force-dependent binding kinetics. However, it is challenging to deliver such nanocarriers while avoiding toxic effects on healthy blood cells, as many possess ligands that adhesively interact with selectins. Herein, we describe a nanostructured surface to capture flowing cancer cells, while preventing human neutrophil adhesion. Microtube surfaces with immobilized halloysite nanotubes (HNTs) and E-selectin functionalized liposomal doxorubicin (ESPEG L-DXR) significantly increased the number of breast adenocarcinoma MCF7 cells captured from flow, yet also significantly reduced the number of captured neutrophils. Neutrophils firmly adhered and projected pseudopods on surfaces coated only with liposomes, while neutrophils adherent to HNT-liposome surfaces maintained a round morphology. Perfusion of both MCF7 cells and neutrophils resulted in primarily cancer cell adhesion to the HNT-liposome surface, and induced significant cancer cell death. This work demonstrates that nanostructured surfaces consisting of HNTs and ES-PEG L-DXR can increase CTC recruitment for chemotherapeutic delivery, while also preventing healthy cell adhesion and uptake of therapeutic intended for CTCs.
\end{abstract}

\section{Introduction}

Hematogenous metastasis typically signals a poor patient prognosis, with over $90 \%$ of cancer deaths attributed to the metastatic spread [1]. For metastasis to occur, cancer cells detach from the primary tumor, invade through the basement membrane, and intravasate into the peripheral circulation as circulating tumor cells (CTCs) [2]. CTCs that express sialylated carbohydrate ligands on their surface can adhesively interact with selectin proteins on the inflamed endothelial cell wall, leading to selectin-mediated CTC tethering and rolling on the endothelium, followed by firm adhesion and arrest [3]. CTCs can then extravasate to the tissue of a distal organ to form secondary metastases, and while the percentage of CTCs that survive this pathway is small $(<0.01 \%)$, the formation of such metastases remains the primary cause of cancer-related deaths $[4,5]$.

Several chemotherapeutics are currently in use for the treatment of cancers, including doxorubicin, which is in use for the treatment of Kaposi's sarcoma, acute leukemia, metastatic breast cancer, and other lymphomas and sarcomas [6]. Doxorubicin is an adriamycin anthracycline antibiotic that can induce cancer cell death via DNA intercalation, inhibition of topoisomerase II, and formation of free radicals $[7,8]$. However, the nonspecific effects of doxorubicin are numerous, and include systemic toxicity, tissue necrosis, neutropenia, and cardiomyopathy $[9,10]$. Nanobiotechnology has contributed to the development of novel drug delivery vehicles to both enhance the efficiency of doxorubicin while reducing its toxic effects, such as polymers, dendrimers, and liposomes [11]. Liposomal doxorubicin (L-DXR) in particular has been shown to enhance overall drug efficacy by altering pharmacokinetics, increasing circulation time, and reducing non-specific toxic effects [12-14]. Polyethylene glycol (PEG) conjugation to the liposome surface provides steric stabilization and increased circulation time of the drug and can also aid in tumor uptake due to enhanced permeability and retention effects $[14,15]$. The efficacy of L-DXR has been shown clinically as evidenced by Doxil, which has been approved by the FDA 
for use in treating Kaposi's sarcoma [16]. However, current L-DXR formulations lack the targeting mechanisms to treat individual CTCs in the bloodstream, due to the rarity of CTCs amongst healthy circulating blood cells. The concentration of CTCs in the bloodstream of patients is approximately one in a million leukocytes [17] or one in a billion of all healthy blood cells [18].

Cell adhesion molecules known as selectins hold promise in targeting drug delivery vehicles to CTCs under physiological shear flow, due to their rapid, force-dependent binding kinetics $[19,20]$. Sialylated carbohydrate ligands are expressed on the surface of many CTCs, which have the ability to bind to selectin proteins on the inflamed endothelium during metastasis [21-23]. Targeting of CTCs in the bloodstream via selectin-mediated adhesion could reduce the probability of metastasis. However, healthy circulating leukocytes are also known to express sialylated carbohydrate ligands on their surface [24]. Thus, strategies to reduce healthy blood cell adhesion are required for selectinmediated targeting of therapies to CTCs.

The development of nanostructured surfaces to enhance the capture of CTCs for chemotherapeutic delivery while preventing healthy cell adhesion has not been yet investigated. Here, we assessed the application of a nanostructured surface consisting of halloysite nanotubes and nanoscale LDXR to increase cancer cell recruitment while preventing leukocyte adhesion.

\section{Materials and Methods}

2.1. Cell Culture. Colorectal adenocarcinoma cell line COLO 205 (ATCC \#CCL-222) and breast adenocarcinoma cell line MCF7 (ATCC no. HTB-22) were purchased from American Type Culture Collection (Manassas, VA, USA). COLO 205 cells were cultured in RPMI 1640 medium supplemented with $10 \%(\mathrm{v} / \mathrm{v})$ fetal bovine serum and $1 \%(\mathrm{v} / \mathrm{v})$ PenStrep, all purchased from Invitrogen (Grand Island, NY, USA). MCF7 cells were cultured in Eagle's Minimum Essential Medium (EMEM) supplemented with $0.01 \mathrm{mg} / \mathrm{mL}$ bovine insulin, $10 \%(\mathrm{v} / \mathrm{v})$ fetal bovine serum, and $1 \%(\mathrm{v} / \mathrm{v})$ PenStrep, all purchased from Invitrogen. All cancer cell lines were incubated under humidified conditions at $37^{\circ} \mathrm{C}$ and $5 \% \mathrm{CO}_{2}$ and were not allowed to exceed $90 \%$ confluence.

2.2. Neutrophil Isolation. Neutrophils were isolated as previously described [25]. Briefly, human peripheral blood was obtained from healthy blood donors after informed consent via venipuncture and collected using sterile sodium heparincontaining tubes (BD Biosciences, San Jose, CA, USA). Neutrophils were isolated from blood by centrifugation at $480 \times \mathrm{g}$ for $50 \mathrm{~min}$ at $23^{\circ} \mathrm{C}$, in a Marathon $8 \mathrm{~K}$ centrifuge (Fisher Scientific, Pittsburgh, PA, USA) using 1-Step Polymorphs (Accurate Chemical \& Scientific Corporation, Westbury, NY, USA). Neutrophils were extracted and washed in $\mathrm{Mg}^{2+}$ and $\mathrm{Ca}^{2+}$-free Hank's balanced salt solution (HBSS), and all remaining red blood cells in the suspension were lysed hypotonically. Neutrophils were resuspended at a concentration of $1.0 \times 10^{6}$ cells $/ \mathrm{mL}$ in HBSS containing $0.5 \%$
HSA, $2 \mathrm{mM} \mathrm{Ca}^{2+}$ and $10 \mathrm{mM}$ HEPES (Invitrogen), buffered to $\mathrm{pH}$ 7.4.

2.3. Liposomal Doxorubicin Synthesis. Nanoscale liposomes were synthesized as described previously [26]. Briefly, lipids were dried overnight and rehydrated using a thin lipid film hydration method $[27,28]$ with $125 \mathrm{mM}$ ammonium sulfate (Sigma-Aldrich, St. Louis, MO, USA). Lipids were then subjected to 10 freeze-thaw cycles and extruded to prepare empty liposomes (EL). Doxorubicin hydrochloride (SigmaAldrich) was encapsulated within ELs using an ammonium sulfate remote loading method, at a doxorubicin-to-lipid ratio of $0.2: 1(\mathrm{w} / \mathrm{w})$. Excess DXR was removed using gel exclusion chromatography with Sephadex G-50 (SigmaAldrich). Liposomal doxorubicin concentration was determined by spectrophotometry $(\lambda=490 \mathrm{~nm})$. The loading efficiency of doxorubicin was determined to be $>95 \%$.

2.4. Targeted Liposome Preparation. E-selectin functionalized L-DXR (ES-PEG L-DXR) and E-selectin functionalized empty liposomes (ES-PEG EL) were prepared using a postinsertion technique [29]. Recombinant human E-selectin/Fc chimera (rhE/Fc) (R\&D Systems, Minneapolis, MN, USA) was thiolated and conjugated to 1,2-distearoyl-sn-glycero3-phosphoethanolamine-N-maleimide 2000 (DSPE-PEG 2000 maleimide) (Avanti Polar Lipids, Alabaster, AL, USA). To incorporate ES-polyethylene glycol (ES-PEG) conjugates into the lipid bilayer, ES-PEG was incubated with liposomes at $50^{\circ} \mathrm{C}$ for $30 \mathrm{~min}$. Liposomes were stored at $4^{\circ} \mathrm{C}$ for $<1$ week until usage.

The mean particle diameter and zeta potential of the liposome formulations were measured by dynamic light scattering, using a Malvern Zetasizer Nano ZS (Malvern Instruments Ltd., Worcestershire, UK) according to the manufacturer's protocols. Mean particle diameter and zeta potential measurements of the liposome formulations were similar to values previously reported [26]. To determine successful E-selectin conjugation to liposome surface, $10 \mu \mathrm{L}$ of fluorescently tagged liposomes were mixed with $490 \mu \mathrm{L}$ of MCF7 cells $\left(10^{6}\right.$ cells $\left./ \mathrm{mL}\right)$ and exposed to shear flow in a cone and plate viscometer at $2.0 \mathrm{dyn} / \mathrm{cm}^{2}$ for $10 \mathrm{~min}$. Fluorescent liposomes adhered to the cell surface were measured using an Accuri C6 flow cytometer (Accuri Cytometers, Inc., Ann Arbor, MI, USA). To assess fluorescent liposome adhesion and internalization to MCF7 cells, sheared samples were incubated for $60 \mathrm{~min}$ at $37^{\circ} \mathrm{C}$ and then imaged using confocal microscopy.

2.5. Confocal Microscopy. Fluorescent ES-conjugated liposomes were used to verify selectin-mediated adhesion to cancer cells. Fluorescent lipids were synthesized using fluorescent BODIPY-cholesterol (Avanti Polar Lipids). Cells were incubated with $2 \mu \mathrm{L}$ of $10 \mathrm{mg} / \mathrm{mL}$ trihydrochloride trihydrate (Hoechst 33342) (Invitrogen, Carlsbad, CA, USA) for 15 minutes to image cell nuclei. Cells were then placed on glass coverslips and visualized with a Zeiss 710 Spectral Confocal Microscope System (Carl Zeiss MicroImaging GmbH, Jena, Germany) at 65x magnification with FITC and DAPI filters. 
Images were processed using Zen 2009 light edition software (Carl Zeiss MicroImaging GmbH).

2.6. MCF7 Static Assays. MCF7 cells were cultured in complete media at a concentration of $1 \times 10^{5}$ cells $/ \mathrm{mL}$ on 12-well plates (Becton Dickinson, Franklin Lakes, NJ, USA). Cells were treated with ES-PEG L-DXR, L-DXR, or EL at volumes of 0 to $5 \mu \mathrm{L}$ for 18 hours. MCF7 cells were treated with Accutase (Sigma-Aldrich) for $5 \mathrm{~min}$ at $37^{\circ} \mathrm{C}$, to remove adherent cells from the surface. Cells were then washed with phosphate buffered saline (PBS) at $1000 \mathrm{rpm}$ in a refrigerated centrifuge (Allegra XX-22R Centrifuge; Beckman Coulter, Brea, CA, USA) and resuspended in fresh media. After 4 days, cell viability was evaluated on a hemocytometer (Hausser Scientific, Horsham, PA, USA) using a trypan blue exclusion assay (Lonza, Wilkersville, MD, USA). Cells were also treated with $0.5 \mu \mathrm{L}$ of liposome solution and assessed for viability over a period of 1-4 days. Cell morphology and uptake of doxorubicin were imaged using brightfield and fluorescence microscopy, respectively.

2.7. Cancer Cell and Neutrophil Preparation for Capture Experiments. MCF7 and COLO 205 cells were treated with Accutase (Sigma, St. Louis, MO, USA) for 5-10 min before handling. MCF7 and COLO 205 cells were washed in PBS and resuspended at a concentration of $1.0 \times 10^{6} \mathrm{cells} / \mathrm{mL}$, in a flow buffer of HBSS containing 0.5\% HSA, $2 \mathrm{mM} \mathrm{Ca}^{2+}$, and $10 \mathrm{mM}$ HEPES (Invitrogen), buffered to $\mathrm{pH}$ 7.4. For neutrophil capture experiments, freshly isolated neutrophils were resuspended in flow buffer at a concentration of $1.0 \times 10^{6}$ cells $/ \mathrm{mL}$. For combined cell capture assays, MCF7 or COLO 205 cells were resuspended in flow buffer with neutrophils at a $1: 1$ cancer cell to neutrophil ratio, at a concentration of $1.0 \times 10^{6}$ cells $/ \mathrm{mL}$.

2.8. HNT-Liposome Surface Functionalization. Microrenathane microtubing (Braintree Scientific, Braintree, MA, USA) of inner diameter $300 \mu \mathrm{m}$ was cut to $55 \mathrm{~cm}$ in length and washed with $75 \%$ ethanol. To prepare HNT-coated microtube surfaces, a $6.6 \%$ by weight HNT solution (NaturalNano, Rochester, NY, USA) was treated via sonication, followed by filtration [26]. Microtubes were washed using distilled water, followed by incubation with $2: 8$ poly-L-lysine $(0.1 \% \mathrm{w} / \mathrm{v}$, Sigma) for $5 \mathrm{~min}$ and then incubation with treated HNT solution for $3 \mathrm{~min}$. Microtubes were washed thoroughly with distilled water to remove excess HNTs in solution and were incubated overnight at RT. Surface immobilization of liposomes was achieved by incubating E-selectin functionalized liposome solution (ES-PEG L-DXR or ES-PEG EL) for 2.5 hours within HNT-coated microtubes. Smooth microtubes were prepared by immobilizing liposomes on the surface in the absence of HNTs. All surfaces were blocked for nonspecific adhesion for $1 \mathrm{~h}$ with $5 \%$ bovine serum albumin (BSA) (Sigma-Aldrich) in PBS (w/v). All incubation steps were preceded and followed by thorough washes with PBS. Immobilized E-selectin proteins were activated prior to cell capture experiments via perfusion of calcium-enriched flow buffer.
2.9. Capture Experiments. To visualize cell adhesion and capture, functionalized microtubes were secured onto the stage of an Olympus IX81 motorized inverted microscope (Olympus, Center Valley, PA, USA). A motorized syringe pump (KDS 230; IITC Life Science, Woodland Hills, CA, USA) was used to perfuse cell suspensions through microtubes at physiologically relevant flow rates. Cancer cells and combined cell solutions were initially perfused through microtubes at a rate of $0.008 \mathrm{~mL} / \mathrm{min}$ (wall shear stress of 0.5 dynes $/ \mathrm{cm}^{2}$ ) for $30 \mathrm{~min}$, and then $0.04 \mathrm{~mL} / \mathrm{min}$ (wall shear stress of 2.5 dynes $/ \mathrm{cm}^{2}$ ) for another $30 \mathrm{~min}$. Isolated neutrophils were perfused at $0.04 \mathrm{~mL} / \mathrm{min}$ for $60 \mathrm{~min}$. Microtubes were washed with calcium-enriched PBS at $1.0 \mathrm{dyn} / \mathrm{cm}^{2}$ to remove all nonadherent cells. Accutase was gently perfused into the microtube and allowed to incubate for $10 \mathrm{~min}$ to detach adherent cells, followed by perfusion of medium for cell collection. For combined cell assays, neutrophils were separated from cancer cell by centrifugation using 1-Step Polymorphs after cell collection. Cells were cultured in 6-well plates in complete media and analyzed for cell viability at day 4 via trypan blue exclusion.

2.10. Data Acquisition. Videos of cell capture experiments were recorded using a microscope-linked Hitachi CCD camera KP-M1AN (Hitachi, Japan) and a Sony DVD Recorder DVO-1000MD (Sony Electronics Inc., San Diego, CA, USA). Video frames were utilized to determine the number of captured cells per $100,000 \mu \mathrm{m}^{2}$. For combined cell experiments, cancer cell and neutrophil capture measurements were determined based on differences in cell diameter.

2.11. Shape Factor Analysis. Brightfield images of neutrophils captured in smooth and HNT-coated tubes were analyzed for morphological changes using a shape factor analysis. Outlines of neutrophils were created from thresholded brightfield images using edge-detection functions in Metamorph software (Universal Imaging Corp., West Chester, PA, USA). Neutrophil morphological changes were determined using a shape factor program in Metamorph, where shape factor is determined by:

$$
\text { shape factor }=\frac{4 \pi A}{P^{2}},
$$

where $P$ is the perimeter and $A$ is the area of the object (neutrophil). Shape factor values close to 1 correspond to a circle, while values approaching 0 represent spindly or dendritic shapes.

2.12. Statistical Analysis. Data sets were plotted and analyzed using Prism 5.0b for Mac OS X (GraphPad software, San Diego, CA, USA). All results were reported as the mean \pm standard error of the mean. A two-tailed paired $t$-test was used for comparisons between two groups. $P$-values less than 0.05 were considered significant.

\section{Results and Discussion}

3.1. ES-PEG L-DXR Targets and Induces Cell Death in MCF7 Breast Cancer Cells. To assess the functionality of E-selectin 


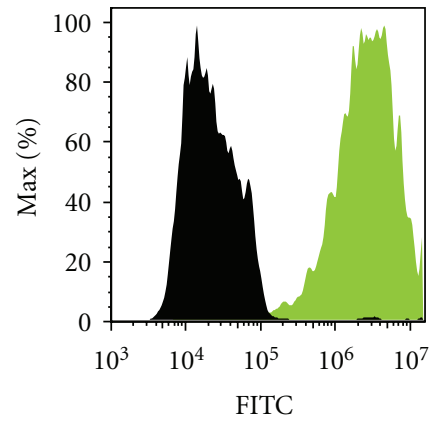

- MCF7 + FL liposomes

$\square$ MCF7 + FL ES liposomes

(a)

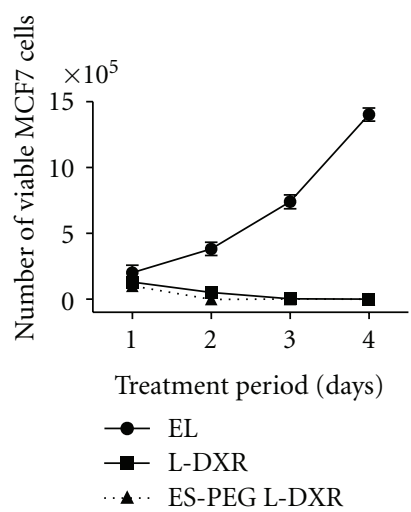

(c)

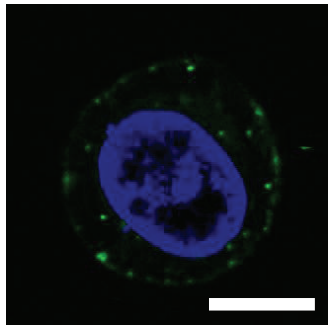

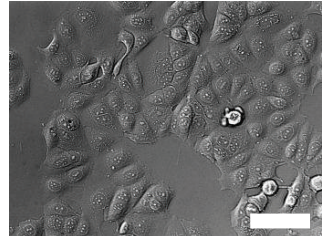

(e)

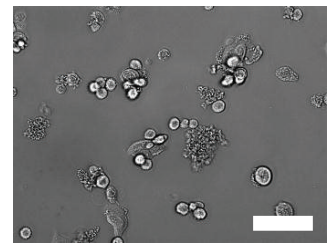

(g)

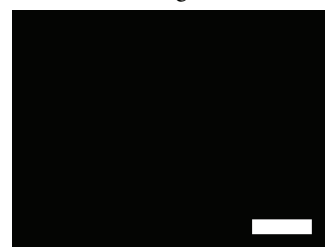

(i)

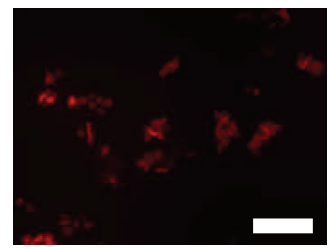

$(\mathrm{k})$

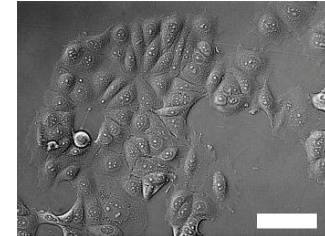

(f)

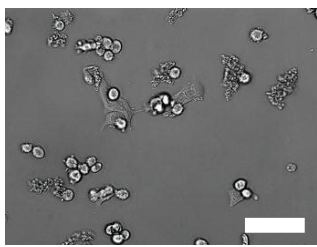

(h)

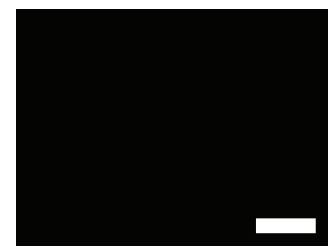

(j)

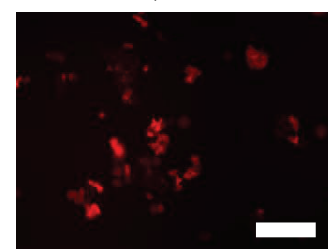

(1)

FIGURE 1: Liposomes functionalized with E-selectin (ES) adhesively bind and deliver doxorubicin to MCF7 breast cancer cells. (a) Flow cytometry fluorescence histograms of MCF7 cells adhered to fluorescently tagged (FL) liposomes and FL ES functionalized liposomes after exposure to fluid shear stress in a cone-and-plate viscometer. FITC: Fluorescein isothiocyanate. (b) Confocal microscopy image of FL ES liposomes (green) bound to MCF7 cells after fluid shear stress exposure. Cell nucleus = blue. Scale bar = 10 $\mu \mathrm{m}$. (c) Number of viable MCF7 cells after treatment with empty liposomes (EL), liposomal doxorubicin (L-DXR) or ES functionalized L-DXR (ES-PEG L-DXR) under static conditions for a period of 1-4 days. (d) Dose response of MCF7 cells after treatment with liposomes over a 4-day period. ((e)-(h)) Bright field microscopy images of untreated MCF7 cells (e) and those treated with EL (f), L-DXR (g), and ES-PEG L-DXR (h) after a 4-day period. Scale bars $=100 \mu \mathrm{m}$. ((i)-(l)) Fluorescence microscopy images of doxorubicin uptake (red) of untreated MCF7 cells (i) and those treated with EL (j), L-DXR (k), and ES-PEG L-DXR (l) after a 4-day period.

conjugated to the liposome surface, fluorescently tagged ESPEG L-DXR was incubated with human breast cancer MCF7 cells and sheared in a cone-and-plate viscometer for $10 \mathrm{~min}$ at $2.0 \mathrm{dyn} / \mathrm{cm}^{2}$. MCF7 cells have been previously shown to roll on ES-coated surfaces, a process that is mediated by CD24 expressed on the MCF7 cell surface [30]. MCF7 cells sheared with fluorescent ES-PEG L-DXR increased in fluorescence by $>99.9 \%$, compared to MCF7 cells sheared with fluorescent liposomes lacking E-selectin (Figure 1(a)). Confocal microscopy revealed that ES-PEG L-DXR not only binds to the cancer cell surface, but also internalizes within the cell over time (Figure 1(b)), making this targeting mechanism well suited for the internalization of ES-PEG LDXR within cancer cells. Static assays verified cellular uptake and cytotoxicity of ES-PEG L-DXR at levels comparable to L-DXR (Figures 1(c) and 1(d)). MCF7 cells treated with either L-DXR (Figure 1(g)) or ES-PEG L-DXR (Figure 1(h)) had observable membrane blebbing and were non-adherent, characteristic of MCF7 cell death. MCF7 cells treated with EL (Figure 1(f)) were adherent and displayed a morphology similar to healthy, untreated MCF7 cells (Figure 1(e)). Fluorescence microscopy confirmed comparable doxorubicin uptake in MCF7 cells treated with L-DXR (Figure 1(k)) and ES-PEG L-DXR (Figure 1(1)), compared to control samples in the absence of doxorubicin (Figures 1(i) and 1(j)). It is apparent from these images that E-selectin adhesion to the cell surface does not hinder internalization and cytotoxic effects of L-DXR on MCF7 cells, making this targeting mechanism suitable for MCF7 treatment under physiological flow.

3.2. Nanostructured Surfaces Enhance MCF7 and COLO 205 Cell Adhesion to ES-PEG L-DXR. Our lab has previously demonstrated that selectin proteins and selectin-coated nanoparticles can be immobilized on the inner surfaces of microtubes for the capture of circulating cells [31], as well as 


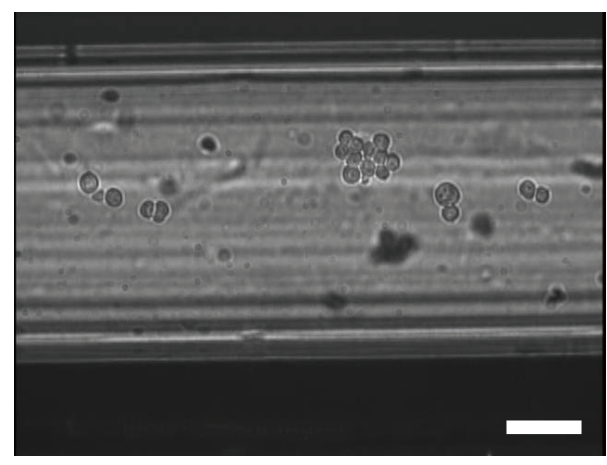

(a)

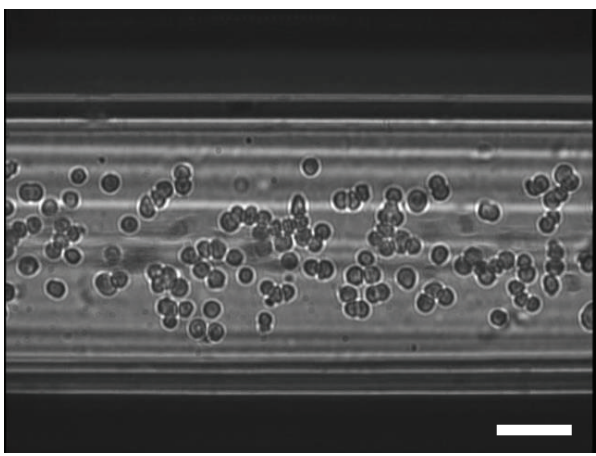

(b)

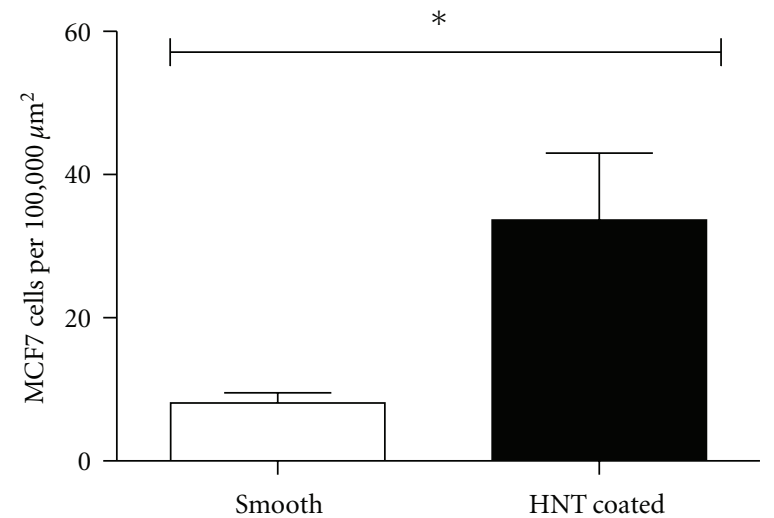

(c)

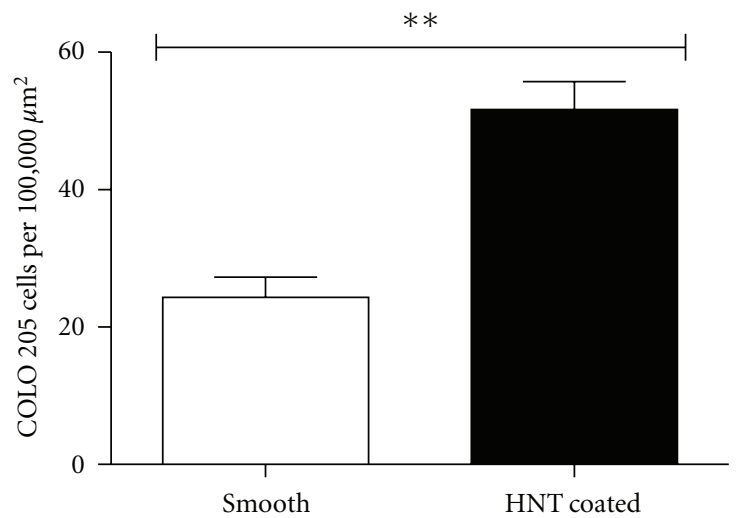

(d)

Figure 2: HNT-liposome-coated surfaces enhance MCF7 and COLO 205 cell capture. (a) MCF7 breast cancer cells adhered to a smooth microtube coated with ES-PEG L-DXR. Scale bar $=100 \mu \mathrm{m}$. (b) MCF7 cells adhered to an HNT-coated microtube with ES-PEG L-DXR. Scale bar $=100 \mu \mathrm{m}$. (c) Number of MCF7 cells adhered to smooth and HNT-coated microtubes per 100,000 $\mu \mathrm{m}^{2}$. (d) Number of COLO 205 cells adhered to smooth and HNT-coated microtubes per $100,000 \mu \mathrm{m}^{2} .{ }^{*} P<0.01 .{ }^{* *} P<0.001$.

for use as in vitro models of the microvasculature to examine the rolling adhesion of leukocytes [32] and cancer cells [33]. In this study, smooth microtubes were coated with only ESPEG L-DXR. HNT-liposome-coated microtubes were coated with HNTs, followed by ES-PEG L-DXR immobilization. MCF7 or COLO 205 cells were perfused through microtubes at shear stresses $\left(0.5-2.5 \mathrm{dyn} / \mathrm{cm}^{2}\right)$ observed in the microvasculature in vivo [34]. Both cell lines were chosen as model CTCs because they express sialylated carbohydrate ligands and exhibit rolling adhesion properties on immobilized Eselectin surfaces [30, 35]. MCF7 cells were captured from flow in both smooth (Figure 2(a)) and HNT-liposomecoated microtubes (Figure 2(b)). However, an increased number of MCF7 cells were captured on HNT-liposome surfaces, compared to smooth surfaces. A significant three fold increase in MCF7 (Figure 2(c)) and two fold increase in COLO 205 cell capture (Figure 2(d)) per 100,000 $\mu \mathrm{m}^{2}$ were observed on HNT-liposome-coated surfaces, compared to smooth surfaces coated with ES-PEG L-DXR. HNTcoated microtubes have been previously shown to increase the adsorption of selectin proteins [36], compared to the adsorption of selectins within microtubes in the absence of HNTs. It is likely that HNT coatings can also enhance the deposition of ES-PEG L-DXR, which could increase the recruitment of flowing cancer cells.

\subsection{Nanostructured Surfaces Reduce Neutrophil Adhesion to} ES-PEG L-DXR. While HNT-coated surfaces can enhance the delivery of chemotherapeutics to CTCs via selectinmediated adhesion, it is also important to examine the effects of such surface interactions with healthy blood cells. Leukocytes, which express sialylated carbohydrate ligands, outnumber CTCs by roughly one million to one in patients, on average [37]. Thus, leukocytes could potentially uptake the majority, if not all of the encapsulated chemotherapeutic being delivered. However, capture assays revealed that the number of neutrophils adhered to HNT-liposome-coated surfaces (Figure 3(b)) was largely diminished compared to smooth surfaces (Figure 3(a)), the opposite of the trend observed in cancer cell capture studies. Similar trends were also observed in the isolation of CTCs from a suspension of mononuclear cells [38]. A significant $>94 \%$ reduction of neutrophils captured per $100,000 \mu \mathrm{m}^{2}$ was confirmed in HNT-liposome-coated microtubes, compared to smooth microtubes coated with ES-PEG L-DXR (Figure 3(c)). 


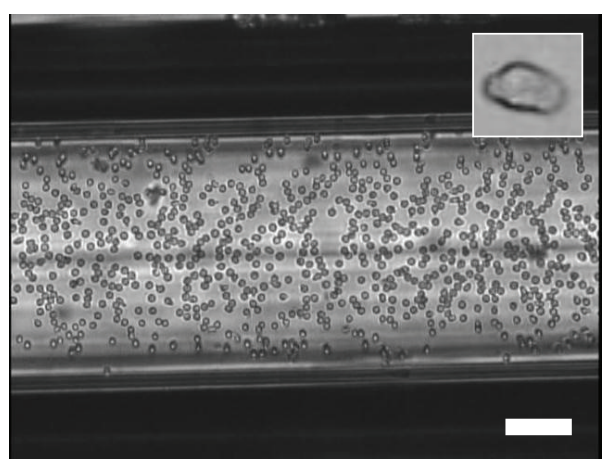

(a)

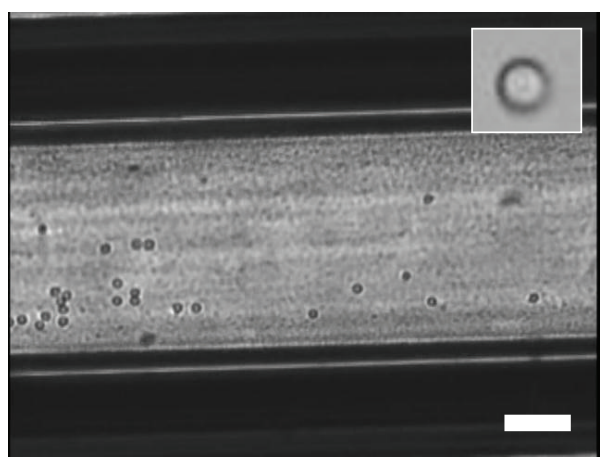

(b)

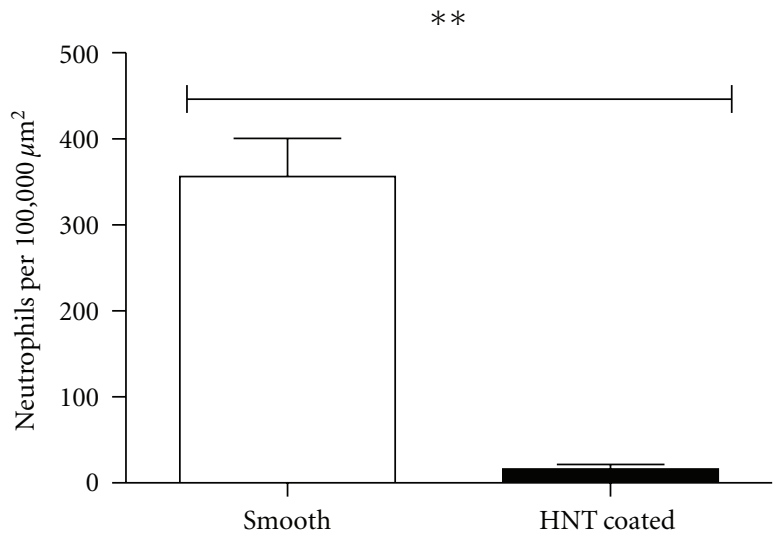

(c)

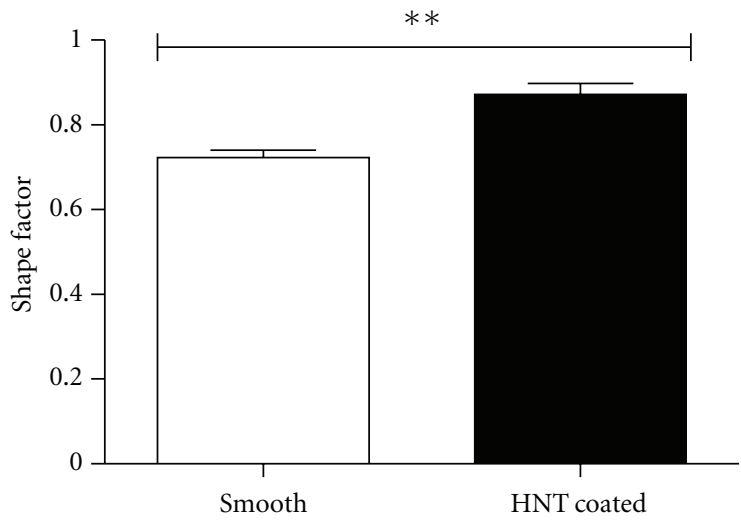

(d)

FIGURE 3: HNT-liposome-coated surfaces reduce and weaken neutrophil adhesion. (a) Neutrophils adhered to a smooth microtube coated with ES-PEG L-DXR. Scale bar $=100 \mu \mathrm{m}$. (b) Neutrophils adhered to an HNT-coated microtube with ES-PEG L-DXR. Scale bar $=100 \mu \mathrm{m}$. Insets show representative cell shapes at higher magnification (approximately $20 \times 20 \mu \mathrm{m}$ ). (c) Number of neutrophils adhered to smooth and HNT-coated microtubes per $100,000 \mu \mathrm{m}^{2}$. (d) Shape factor analysis of neutrophils adhered to smooth and HNT-coated microtubes. ** $P<0.001$.

Cytoskeletal projections known as pseudopods were observed consistently on neutrophils adhered to smooth surfaces, characteristic of firmly adhered and activated neutrophils (Figure 3(a)) [24]. Neutrophils on HNT-liposomecoated surfaces displayed a rounder morphology, characteristic of weaker adhesion of resting neutrophils (Figure 3(b)). Shape factor analysis has previously been utilized to assess neutrophil morphological changes [26] and was performed in this study to determine the effect of adhesion to HNTliposome-coated surfaces on morphological changes. On a scale of 0 (elongated shapes) to 1 (circularity), shape factor analysis verified a significantly higher shape factor in neutrophils adhered to HNT-liposome-coated surfaces (Figure 3(d)), compared to those adhered to smooth surfaces, adding further support that neutrophils display weaker adhesion on HNT-liposome-coated surfaces.

To examine if the reduction in neutrophil adhesion is due to prevention of ES-PEG L-DXR adsorption to the surface, ES-PEG L-DXR was fluorescently tagged and immobilized on smooth and HNT-coated microtubes. However, fluorescence microscopy confirmed that ES-PEG LDXR immobilized within both smooth (Figure 4(b)) and
HNT-coated microtubes (Figure 4(c)), compared to control microtubes in the absence of fluorescent ES-PEG L-DXR (Figure 4(a)). Thus, the reduction in neutrophil adhesion could not be caused by prevention of ES-PEG L-DXR adsorption.

\subsection{HNT-Liposome Surfaces Capture Cancer Cells and Repel} Neutrophils from a Mixture of Cells. The use of HNTliposome-coated surfaces for CTC capture from a combined cell suspension was assessed via perfusion of a $1: 1$ mixture of cancer cells and neutrophils over liposome-coated surfaces. Neutrophils and MCF7 cells were identified based on size; cells $15 \mu \mathrm{m}$ in diameter or less were identified as neutrophils, while cells greater than $15 \mu \mathrm{m}$ were identified as MCF7 or COLO 205 cells. Upon perfusion of neutrophils and MCF7 cells, the number of neutrophils captured decreased on HNT-liposome-coated surfaces (Figure 5(b)) compared to smooth surfaces (Figure 5(a)). Simultaneously, an increase in MCF7 cell capture was observed on HNT-liposomecoated surfaces (Figure 5(b)), compared to smooth surfaces (Figure 5(a)). The number of both MCF7 (Figure 5(c)) and COLO 205 (Figure 5(d)) cells captured was significantly 


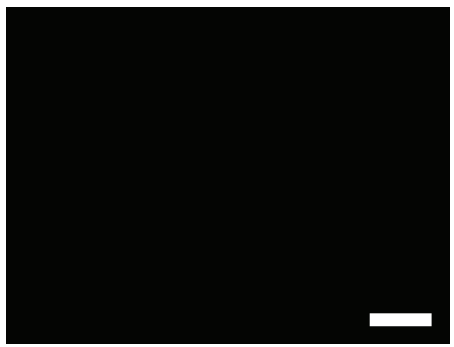

(a)

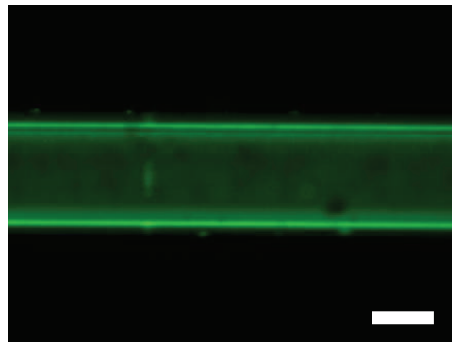

(b)

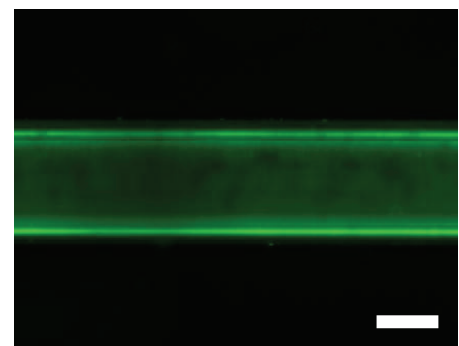

(c)

FIgURE 4: E-selectin functionalized liposomes immobilize on both smooth and HNT-coated microtube surfaces. ((a)-(c)) Fluorescence microscopy images of microtubes coated with BSA (a), smooth microtubes coated with fluorescent ES-PEG L-DXR (b), and HNT-coated microtubes with immobilized fluorescent ES-PEG L-DXR (c). Scale bar $=100 \mu \mathrm{m}$.

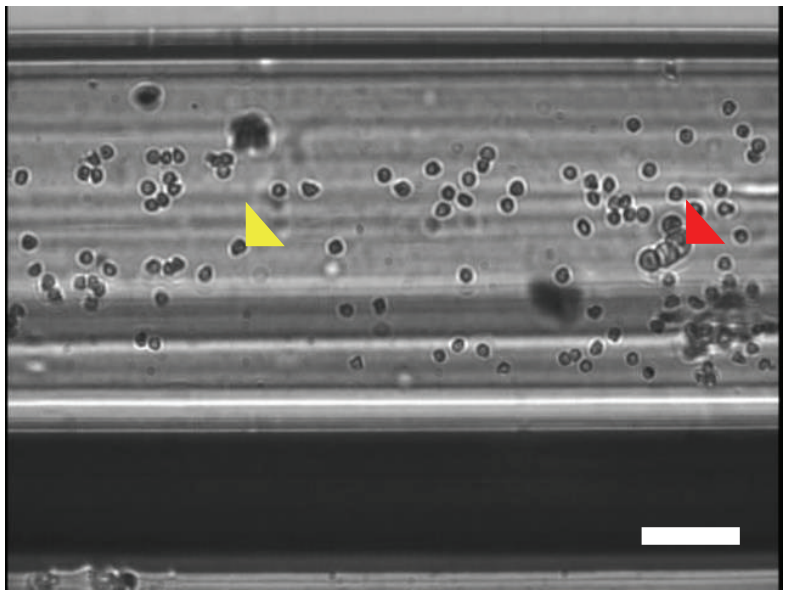

(a)

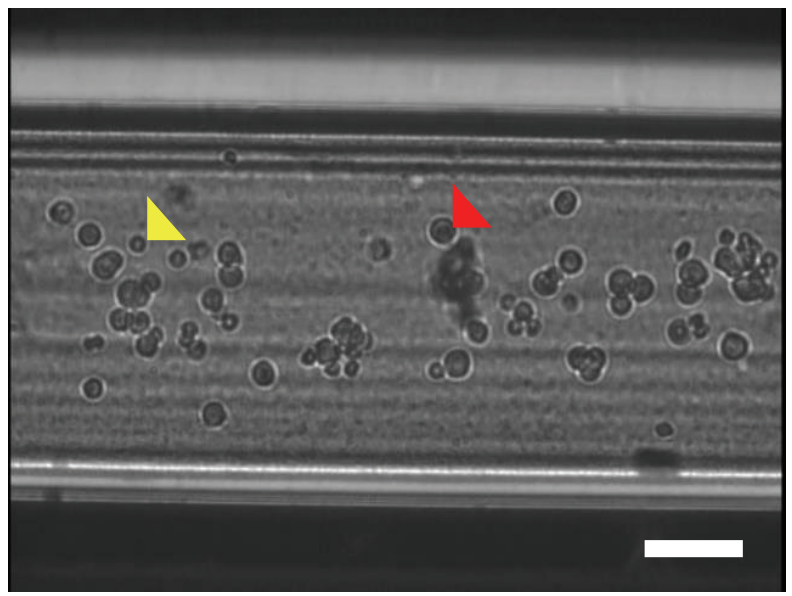

(b)

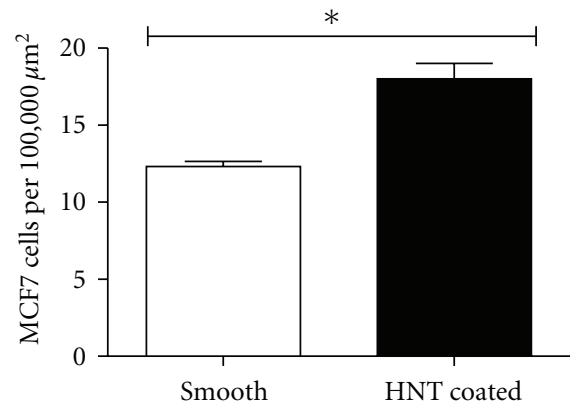

(c)

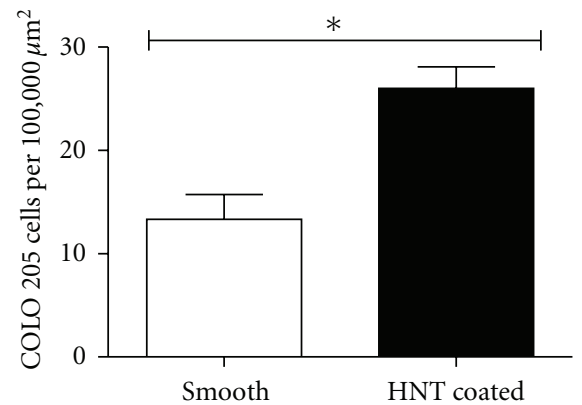

(d)

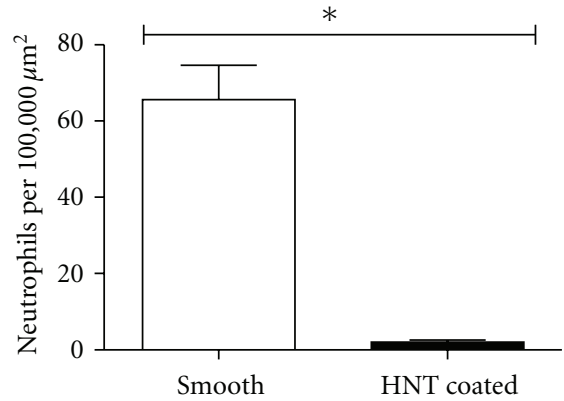

(e)

FIgURE 5: HNT-liposome surfaces simultaneously enhance MCF7 and COLO 205 cell adhesion and reduce neutrophil adhesion from a combined suspension of cancer cells and neutrophils. (a) MCF7 cells (red arrow) and neutrophils (yellow arrow) adhered to a smooth microtube coated with ES-PEG L-DXR. Scale bar $=100 \mu \mathrm{m}$. (b) MCF7 cells (red arrow) and neutrophils (yellow arrow) adhered to an HNTcoated microtube with immobilized ES-PEG L-DXR. Scale bar $=100 \mu \mathrm{m}$. (c) Number of MCF7 cells adhered to smooth and HNT-coated microtubes per $100,000 \mu \mathrm{m}^{2}$. (d) Number of COLO 205 cells adhered to smooth and HNT-coated microtubes per 100,000 $\mu \mathrm{m}^{2}$. (e) Number of neutrophils adhered to smooth and HNT-coated microtubes per 100,000 $\mu \mathrm{m}^{2}{ }^{*} P<0.01$. 


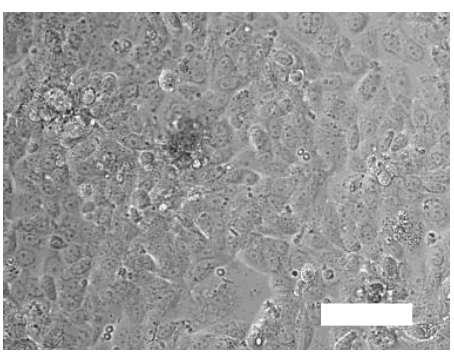

(a)

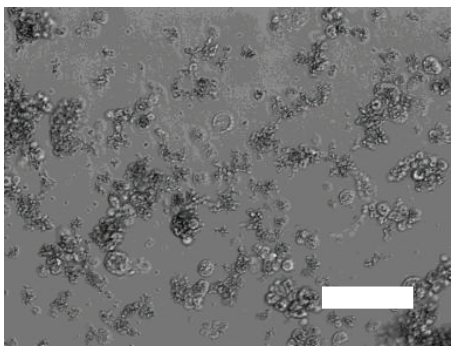

(b)

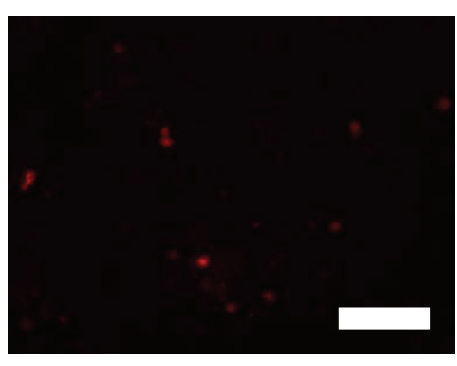

(c)

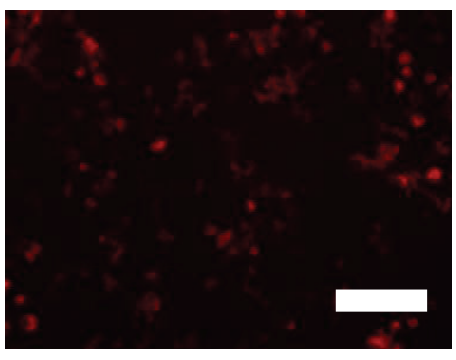

(d)

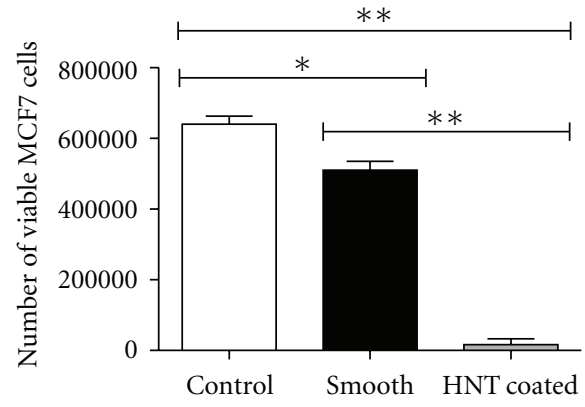

(e)

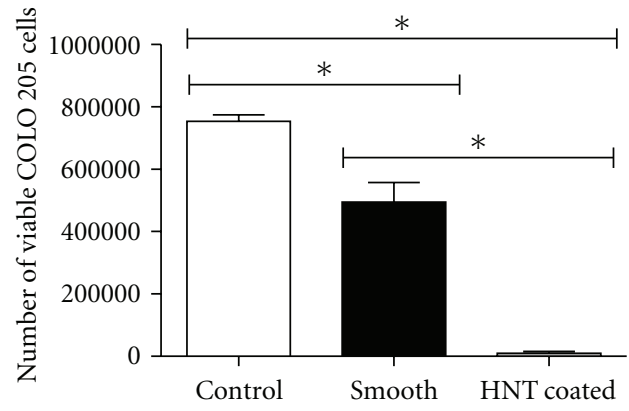

(f)

FIGURE 6: HNT-liposome-coated surfaces successfully deliver doxorubicin to cancer cells from a combined solution of cancer cells and neutrophils. Brightfield images of MCF7 cells captured on a smooth surface (a) and an HNT-liposome-coated surface (b), from a combined solution of cancer cells and neutrophils. Internalization of doxorubicin (red) in MCF7 cells captured on a smooth surface (c) and an HNTliposome-coated surface (d), from a combined solution of cancer cells and neutrophils. All images were taken after 4-day incubation at $37^{\circ} \mathrm{C}$. Scale bar $=100 \mu \mathrm{m}$. Number of viable MCF7 (e) and COLO 205 (f) cells after capture on smooth and HNT-liposome-coated surfaces. Control samples represent viable cells captured by ES-PEG EL. ${ }^{*} P<0.01$. ${ }^{* *} P<0.001$.

increased on HNT-liposome-coated surfaces, with significant reductions in neutrophil capture (Figure 5(e)).

\subsection{HNT-Liposome Surfaces Induce Cancer Cell Death within} a Mixture of Cancer Cells and Neutrophils. The ability to successfully deliver doxorubicin to cancer cells within a combined cell suspension was assessed by the removal and collection of captured cells from surfaces, separation of cancer cells from neutrophils, and placement into culture for viability tests. Neutrophils, which have a short lifespan in vivo and typically undergo cell death in vitro within 1828 hours after isolation [39], showed negligible differences in viability whether captured within microtubes or placed directly into culture upon isolation (not shown). After a 4day incubation, brightfield microscopy confirmed that while MCF7 cells captured on smooth surfaces were adherent and displayed healthy morphology (Figure 6(a)). However, MCF7 cells captured on HNT-liposome-coated surfaces were non-adherent and displayed significant membrane blebbing characteristic of apoptosis (Figure 6(b)). MCF7 cells captured on smooth surfaces exhibited minimal doxorubicin uptake based on fluorescence images (Figure 6(c)), while MCF7 cells captured on HNT-liposome-coated surfaces displayed increased doxorubicin uptake (Figure 6(d)). Cell viability assays confirmed doxorubicin-induced cell death, with a significant decrease in MCF7 (Figure 6(e)) and COLO 205 cell viability (Figure 6(f)) when captured on smooth tubes coated with ES-PEG L-DXR, compared to control smooth surfaces coated with ES-PEG EL. However, the number of viable MCF7 and COLO 205 cells captured on HNT-liposome-coated surfaces was significantly reduced, compared to the number of viable cells captured on smooth surfaces. Similar trends were observed previously in KG-1a and COLO 205 cells captured from a solution consisting only of cancer cells [26]. These results demonstrate that a combined surface of HNTs and ES-PEG L-DXR can serve a dual role in: (1) delivering chemotherapeutics to CTCs and (2) reducing leukocyte adhesion and interaction with chemotherapeutics and thus reducing toxic side effects on healthy blood cells.

\section{Conclusion}

Here, we have demonstrated a technique utilizing cell adhesion molecules to deliver therapeutics to CTCs while actively preventing blood cell adhesion. ES-PEG L-DXR was shown to adhesively interact with MCF7 breast cancer cells and induce cell death. Under physiological flow rates, HNTliposome-coated surfaces significantly increased the number of captured MCF7 and COLO 205 cancer cells, compared to smooth surfaces coated with ES-PEG L-DXR. The opposite trend occurred with healthy blood cells, as perfusion of neutrophils over HNT-liposome coated surfaces significantly reduced the number of captured cells. In a combined 
solution of cancer cells and neutrophils, HNT-liposomecoated surfaces simultaneously increased the number of MCF7 and COLO 205 cells captured, while significantly reducing neutrophil capture to minimum levels. Liposomal doxorubicin was successfully delivered to MCF7 and COLO 205 cells in flow assays with a combined solution of cancer cells and neutrophils. The application of HNT-liposome surfaces can enhance chemotherapeutic delivery to CTCs and reduce the probability of metastasis. Additionally, the unique ability of such surfaces to prevent normal blood cell interactions can reduce toxic non-specific effects and dramatically reduce chemotherapeutic dosages required for CTC treatment.

\section{Acknowledgments}

This work was supported by the National Institutes of Health, Grant no. CA143876. The authors acknowledge Jeff Mattison for work with blood sample collection and donor recruitment.

\section{References}

[1] C. L. Chaffer and R. A. Weinberg, "A perspective on cancer cell metastasis," Science, vol. 331, no. 6024, pp. 1559-1564, 2011.

[2] A. D. Hughes and M. R. King, "Nanobiotechnology for the capture and manipulation of circulating tumor cells," WIREs Nanomedicine and Nanobiotechnology, vol. 4, no. 3, pp. 291309, 2011.

[3] L. M. Coussens and Z. Werb, "Inflammation and cancer," Nature, vol. 420, no. 6917, pp. 860-867, 2002.

[4] A. F. Chambers, G. N. Naumov, S. A. Vantyghem, and A. B. Tuck, "Molecular biology of breast cancer metastasis. Clinical implications of experimental studies on metastatic inefficiency," Breast Cancer Research, vol. 2, no. 6, pp. 400-407, 2000.

[5] I. C. MacDonald, A. C. Groom, and A. F. Chambers, "Cancer spread and micrometastasis development: quantitative approaches for in vivo models," BioEssays, vol. 24, no. 10, pp. 885-893, 2002.

[6] R. C. Young, R. F. Ozols, and C. E. Myers, "The anthracycline antineoplastic drugs," The New England Journal of Medicine, vol. 305, no. 3, pp. 139-153, 1981.

[7] J. Bouma, J. H. Beijnen, A. Bult, and W. J. M. Underberg, "Anthracycline antitumour agents. A review of physicochemical, analytical and stability properties," Pharmaceutisch Weekblad, vol. 8, no. 3, pp. 109-133, 1986.

[8] N. Osheroff, A. H. Corbett, and M. J. Robinson, "Mechanism of action of topoisomerase II-targeted antineoplastic drugs," Advances in Pharmacology, vol. 29, pp. 105-126, 1994.

[9] L. H. Reddy, "Drug delivery to tumours: recent strategies," Journal of Pharmacy and Pharmacology, vol. 57, no. 10, pp. 1231-1242, 2005.

[10] A. Fritze, F. Hens, A. Kimpfler, R. Schubert, and R. PeschkaSüss, "Remote loading of doxorubicin into liposomes driven by a transmembrane phosphate gradient," Biochimica et Biophysica Acta, vol. 1758, no. 10, pp. 1633-1640, 2006.

[11] M. Saad, O. B. Garbuzenko, E. Ber et al., "Receptor targeted polymers, dendrimers, liposomes: which nanocarrier is the most efficient for tumor-specific treatment and imaging?" Journal of Controlled Release, vol. 130, no. 2, pp. 107-114, 2008.
[12] E. H. Herman, A. Rahman, and V. J. Ferrans, "Prevention of chronic doxorubicin cardiotoxicity in beagles by liposomal encapsulation," Cancer Research, vol. 43, no. 11, pp. 54275432, 1983.

[13] R. Krishna, N. McIntosh, K. W. Riggs, and L. D. Mayer, "Doxorubicin encapsulated in sterically stabilized liposomes exhibits renal and biliary clearance properties that are independent of valspodar (PSC 833) under conditions that significantly inhibit nonencapsulated drug excretion," Clinical Cancer Research, vol. 5, no. 10, pp. 2939-2947, 1999.

[14] A. Gabizon, H. Shmeeda, and Y. Barenholz, "Pharmacokinetics of pegylated liposomal doxorubicin: review of animal and human studies," Clinical Pharmacokinetics, vol. 42, no. 5, pp. 419-436, 2003.

[15] H. D. Han, A. Lee, T. Hwang et al., "Enhanced circulation time and antitumor activity of doxorubicin by comblike polymerincorporated liposomes," Journal of Controlled Release, vol. 120, no. 3, pp. 161-168, 2007.

[16] B. Čeh, M. Winterhalter, P. M. Frederik, J. J. Vallner, and D. D. Lasic, "Stealth liposomes: from theory to product," Advanced Drug Delivery Reviews, vol. 24, no. 2-3, pp. 165-177, 1997.

[17] W. J. Allard, J. Matera, M. C. Miller et al., "Tumor cells circulate in the peripheral blood of all major carcinomas but not in healthy subjects or patients with nonmalignant diseases," Clinical Cancer Research, vol. 10, no. 20, pp. 68976904, 2004.

[18] S. Maheswaran and D. A. Haber, "Circulating tumor cells: a window into cancer biology and metastasis," Current Opinion in Genetics and Development, vol. 20, no. 1, pp. 96-99, 2010.

[19] G. S. Kansas, "Selectins and their ligands: current concepts and controversies," Blood, vol. 88, no. 9, pp. 3259-3287, 1996.

[20] J. Li and M. R. King, "Adhesion receptors as therapeutic targets for circulating tumor cells," Frontiers in Oncology, vol. 2, article 79, 2012.

[21] H. Läubli and L. Borsig, "Selectins promote tumor metastasis," Seminars in Cancer Biology, vol. 20, no. 3, pp. 169-177, 2010.

[22] T. Krause and G. A. Turner, "Are selectins involved in metastasis?" Clinical and Experimental Metastasis, vol. 17, no. 3, pp. 183-192, 1999.

[23] S. Gout, P. L. Tremblay, and J. Huot, "Selectins and selectin ligands in extravasation of cancer cells and organ selectivity of metastasis," Clinical and Experimental Metastasis, vol. 25, no. 4, pp. 335-344, 2008.

[24] T. A. Springer, "Traffic signals for lymphocyte recirculation and leukocyte emigration: the multistep paradigm," Cell, vol. 76, no. 2, pp. 301-314, 1994.

[25] M. J. Mitchell and M. R. King, "Shear-induced resistance to neutrophil activation via the formyl peptide receptor," Biophysical Journal, vol. 102, no. 8, pp. 1804-1814, 2012.

[26] M. J. Mitchell, C. S. Chen, V. Ponmudi, A. D. Hughes, and M. R. King, "E-selectin liposomal and nanotube-targeted delivery of doxorubicin to circulating tumor cells," Journal of Controlled Release, vol. 160, no. 3, pp. 609-617, 2012.

[27] S. Amselem, R. Cohen, S. Druckmann et al., "Preparation and characterization of liposomal doxorubicin for human use," Journal of Liposome Research, vol. 2, no. 1, pp. 93-123, 1992.

[28] G. Haran, R. Cohen, L. K. Bar, and Y. Barenholz, "Transmembrane ammonium sulfate gradients in liposomes produce efficient and stable entrapment of amphipathic weak bases," Biochimica et Biophysica Acta, vol. 1151, no. 2, pp. 201-215, 1993.

[29] A. N. Lukyanov, T. A. Elbayoumi, A. R. Chakilam, and V. P. Torchilin, "Tumor-targeted liposomes: doxorubicinloaded long-circulating liposomes modified with anti-cancer 
antibody," Journal of Controlled Release, vol. 100, no. 1, pp. 135-144, 2004.

[30] J. H. Myung, K. A. Gajjar, R. M. Pearson, C. A. Launiere, D. T. Eddington, and S. Hong, "Direct measurements on CD24mediated rolling of human breast cancer MCF-7 cells on Eselectin," Analytical Chemistry, vol. 83, no. 3, pp. 1078-1083, 2011.

[31] Z. Huang and M. R. King, "An immobilized nanoparticlebased platform for efficient gene knockdown of targeted cells in the circulation," Gene Therapy, vol. 16, no. 10, pp. 12711282, 2009.

[32] D. Lee, J. B. Schultz, P. A. Knauf, and M. R. King, "Mechanical shedding of L-selectin from the neutrophil surface during rolling on sialyl lewis $\mathrm{x}$ under flow," The Journal of Biological Chemistry, vol. 282, no. 7, pp. 4812-4820, 2007.

[33] X. Yin, K. Rana, V. Ponmudi, and M. R. King, "Knockdown of fucosyltransferase III disrupts the adhesion of circulating cancer cells to E-selectin without affecting hematopoietic cell adhesion," Carbohydrate Research, vol. 345, no. 16, pp. 23342342, 2010.

[34] M. B. Kim and I. H. Sarelius, "Distributions of wall shear stress in venular convergences of mouse cremaster muscle," Microcirculation, vol. 10, no. 2, pp. 167-178, 2003.

[35] K. Rana, C. A. Reinhart-King, and M. R. King, "Inducing apoptosis in rolling cancer cells: a combined therapy with aspirin and immobilized TRAIL and E-selectin," Molecular Pharmaceutics, vol. 9, Article ID 120702, 2012.

[36] A. D. Hughes and M. R. King, "Use of naturally occurring halloysite nanotubes for enhanced capture of flowing cells," Langmuir, vol. 26, no. 14, pp. 12155-12164, 2010.

[37] B. T. Greene, A. D. Hughes, and M. R. King, "Circulating tumor cells: the substrate of personalized medicine?" Frontiers in Oncology, vol. 2, article 69, 2012.

[38] A. D. Hughes, J. Mattison, L. T. Western, J. D. Powderly, B. T. Greene, and M. R. King, "Microtube device for selectinmediated capture of viable circulating tumor cells from blood," Clinical Chemistry, vol. 58, no. 5, pp. 846-853, 2012.

[39] C. G. Begley, A. F. Lopez, N. A. Nicola et al., "Purified colonystimulating factors enhance the survival of human neutrophils and eosinophils in vitro: a rapid and sensitive microassay for colony-stimulating factors," Blood, vol. 68, no. 1, pp. 162-166, 1986. 

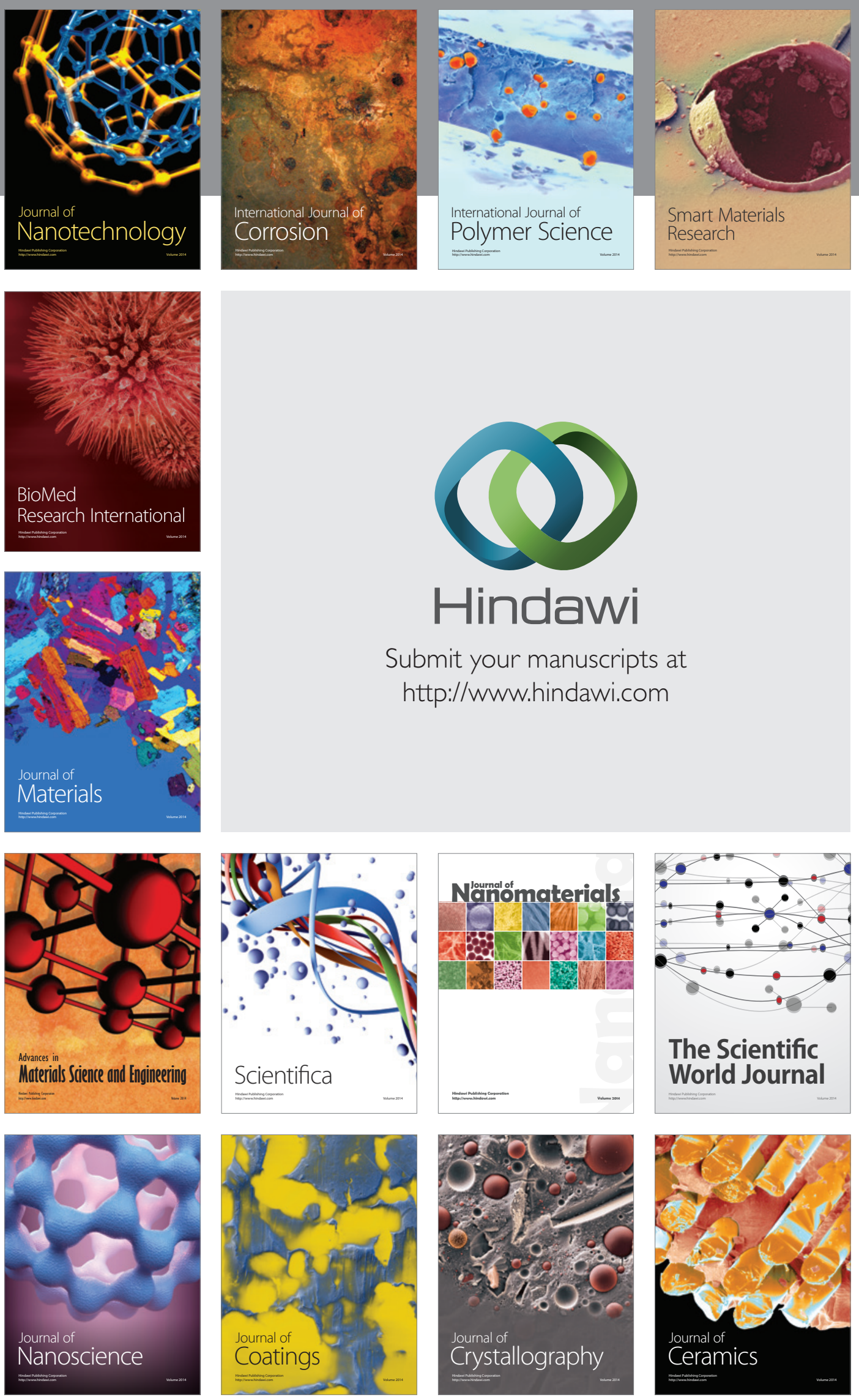

The Scientific World Journal

Submit your manuscripts at

http://www.hindawi.com

\section{World Journal}

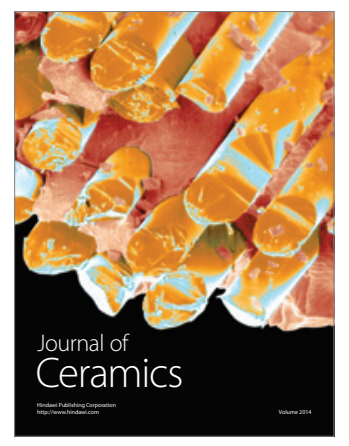

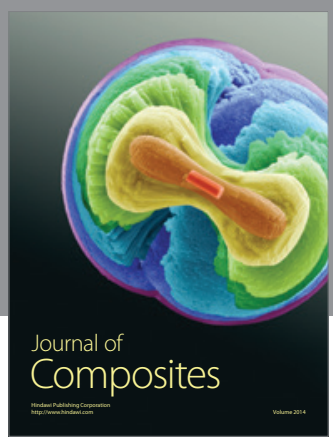
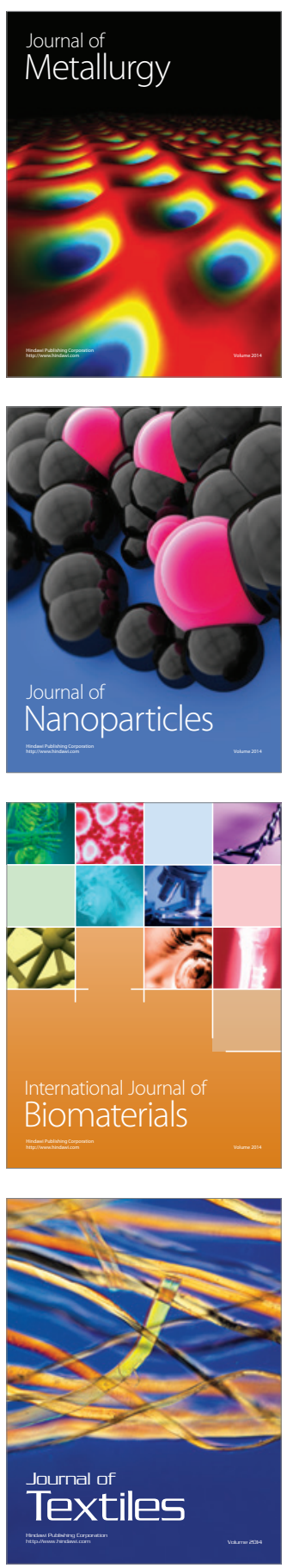